\title{
Improvement of Spatial Resolution for Nonlinear Raman Microscopy by Spatial Light Modulation
}

\author{
Motohiro BANNo, Konosuke ONDA, and Hiroharu YUI ${ }^{\dagger}$ \\ Department of Chemistry, Faculty of Science, Tokyo University of Science, 1-3 Kagurazaka, Shinjuku, \\ Tokyo 162-8601, Japan
}

\begin{abstract}
The development of a stimulated Raman scattering (SRS) microscope with a wavefront modulation unit is presented. In the apparatus, two beams for introducing the SRS process were focused into the sample with an objective lens. In the pathway of the Stokes beam, which is one of the two incident beams, a spatial light modulator (SLM) was located. Using the SLM, the wavefront of the Stokes beam was modulated to make the shape of the focal point a concentric circular pattern. By this spot shaping technique, the area where the SRS signal generates is restricted. The instrument response function (IRF) of the SRS microscope was examined by measuring the SRS intensity while scanning the sample position. From the result, the width of the IRF was reduced by about $15 \%$ by the wavefront modulation. It is suggested that the introduction of SLM is a way to improve the IRF of vibrational spectroscopic microscopes.
\end{abstract}

Keywords Microscopy, Raman spectroscopy, nonlinear spectroscopy, super-resolution, wavefront, spatial light modulation

(Received August 30, 2016; Accepted September 30, 2016; Published January 10, 2017)

\section{Introduction}

Microspectroscopy is applied in many fields, such as the biological, medical, and material fields. ${ }^{1-5}$ In this method, an incident light is focused into a part with the diameter of several microns, and optical responses, such as absorption and scattering, from the part are recorded. Because the optical responses are molecular-selective, the spatial distribution of the target molecule can be visualized while scanning the position of the focal point in the sample.

Among many spectroscopic methods, vibrational spectroscopy has several advantages for application to microspectroscopy. First, the wavenumbers of the normal vibrational modes change sensitively depending on the structures of the molecules. By analyzing the vibrational spectrum, the species and the structures of molecules can be identified. Second, the peak positions and the widths of the bands due to the vibrational transitions sensitively reflect the microscopic environments of the molecules, such as the solute-solvent interactions in solutions. When vibrational spectroscopy is combined with the microscopy, the spatial inhomogeneity of the chemical species distributions in the sample can be observed. Finally, because any multiatomic molecule exhibits vibrational bands, no staining reagent is necessary for vibrational spectroscopic measurements.

Infrared (IR) spectroscopy is one of the popular vibrational spectroscopic methods. When IR spectroscopy is combined with microscopy, it is expected that vibrational microspectroscopy is realized. However, IR light is readily absorbed by water. Therefore, IR microspectroscopy is seldom applied for the imaging of biological samples. It is also disadvantageous that

† To whom correspondence should be addressed.

E-mail: yui@rs.kagu.tus.ac.jp the spatial resolution is limited to several to tens of microns due to the long wavelength of the IR light. ${ }^{6}$

Instead, it is possible to combine Raman spectroscopy with microscopy. In Raman spectroscopy, the wavelength of the light for the excitation of a sample can be arranged. The spatial resolution for Raman microspectroscopy is determined with the diffraction limit of the incident excitation light. ${ }^{7}$ When coherent anti-Stokes Raman scattering (CARS), which is a nonlinear Raman process, is utilized, the spatial resolution of $300 \mathrm{~nm}$ has been achieved. ${ }^{8}$ By combining Raman spectroscopy with microscopy, imaging based on the vibrational spectroscopic signal with a higher spatial resolution should be realized.

It is also possible that near-IR light is used for the excitation of Raman microspectroscopy. Near-IR light is less scattered by media due to the long wavelength. ${ }^{7}$ It is known that interferences from fluorescence is substantially reduced when near-IR light; instead of visible light, is used for the excitation of the Raman process. ${ }^{9}$ Therefore, by applying the near-IR light for the excitation of the Raman process in Raman microspectroscopy, the Raman imaging of samples deeply buried by light-scattering media with little interference from fluorescence should be realized. However, when near-IR light is utilized, the spatial resolution becomes somewhat poor due to the wavelength longer than the visible light. To apply near-IR light to Raman microspectroscopy more effectively, it is essential to improve the spatial resolution.

One method to improve the spatial resolution is to apply nonlinear Raman spectroscopy. As mentioned above, when CARS is utilized, the spatial resolution is improved by a factor of about two. ${ }^{8}$ This is because the signals from the nonlinear Raman processes generate from only where two beams introducing the nonlinear Raman process overlap spatially, and the amplitudes of the electric fields are more than the thresholds for the signal generation. ${ }^{5}$ 


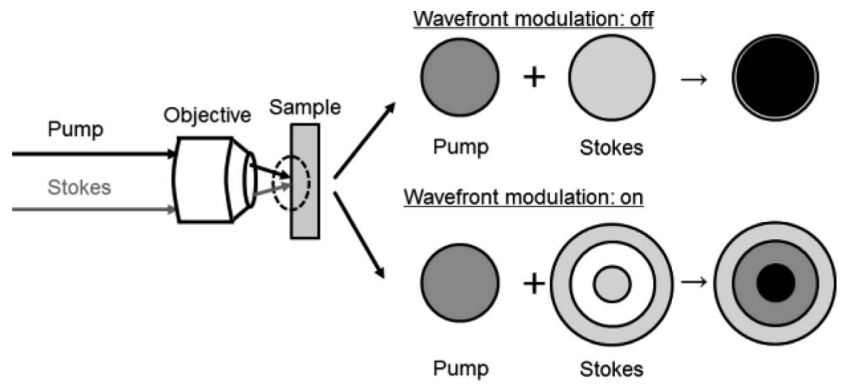

Fig. 1 Restriction of the area generating the SRS signal. In the right side, the images of the focal points for the pump and Stokes beams are shown. When the wavefront modulation is off, the SRS signal generates from almost whole of the focal points (black circle in the upper side). When the wavefront modulation is on, the signal generates only from the area indicated with the small black circle in the bottom side.

We have developed a near-IR stimulated Raman scattering (SRS) microscope. ${ }^{18}$ In the apparatus, the SRS process is applied to avoid the contributions from the non-resonant background observed in CARS spectroscopy. ${ }^{19}$ The apparatus can be applied for the measurements of deeply buried samples. However, the spatial resolution is less than the microscopes in which UV-VIS lights are utilized. We have combined the SRS microscope with the light interferometry to improve the depth resolution. ${ }^{18}$ However, the lateral resolution is not improved with the technique, and the poor lateral spatial resolution still remains.

To overcome the difficulty, we adapted the wavefront modulation technique. Based on the Fourier optics, when a beam is focused by a lens, the spot shape at the focal point is related to the wavefront of the beam spot before focusing by the two-dimensional complex Fourier transform. ${ }^{10}$ Therefore, by modulating the amplitude and the phase of the beam spot before focusing by lens, the spot shape of the focal point can be arbitrarily changed. By far, the wavefront modulation methods have been applied for the improvement of the spatial resolution for fluorescence spectroscopy ${ }^{11-16}$ and pump-dump-probe spectroscopy. ${ }^{17}$ For example, stimulated emission depletion (STED) has been developed. ${ }^{11}$ In this method, it is essential to change the shape of the focal point for the beam for the stimulated emission to a "doughnut" pattern.

In this study, we combine the SRS microscope with the wavefront modulation technique. The nonlinear Raman signal generates from where the electric fields of the two beams introducing the nonlinear Raman process spatially overlap. When the shape of the focal point for one of the two beams is changed to a concentric circular pattern, as shown in Fig. 1, the area where the nonlinear Raman signal generates is restricted to the small center circle. In the present article, we present the development of an SRS microscope capable of wavefront modulation. In the developed apparatus, the wavefront is modulated by a liquid crystal spatial light modulator (SLM). The wavefront pattern for the modulation to make the concentric circular pattern in the focal point was calculated with the iterative Fourier transform. ${ }^{10}$ The improvement of the instrument response function (IRF) by the wavefront modulation was examined. The IRF represents the size of the area generating the SRS signal. Therefore, the width of the IRF correlates strongly with the spatial resolution, which is the minimum distance between two objects being separately observed. As a result, we have successfully developed an SRS microscope with wavefront modulation. This is the first development of the

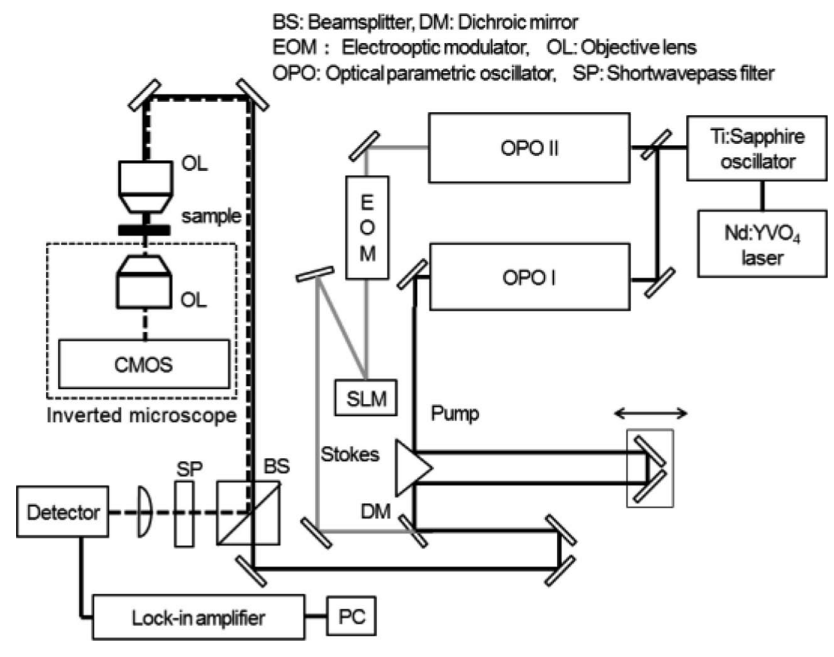

Fig. 2 Schematic diagram of the developed SRS microscope with the SLM.

vibrational spectroscopic microscope with the wavefront modulation technique.

\section{Experimental}

We introduced an SLM to an SRS microscope we had developed. The detail of the SRS microscope has been reported elsewhere. ${ }^{18}$ The block diagram is shown in Fig. 2. Briefly, an output from a Ti:sapphire oscillator (wavelength: $800 \mathrm{~nm}$, power: $3.7 \mathrm{~W}$, pulse duration: $2 \mathrm{ps}$, repetition rate: $76 \mathrm{MHz}$ ) was divided into two, and was used for excitations of two optical parametric amplifiers (OPO I and OPO II). The outputs from OPO I and OPO II were used as the pump and Stokes lights for introducing the SRS process. The amplitude of the Stokes light was modulated with the frequency of $3 \mathrm{MHz}$ by an electro-optic modulator (EOM). After passing through the EOM, the Stokes light was reflected by the SLM. The wavefront of the Stokes light was modulated by the SLM (X10468-08, Hamamatsu Photonics). The SLM was controlled by a personal computer (PC). The relative optical path length between the pump and Stokes lights was scanned by a mechanical delay stage. The powers of the pump and Stokes light at the sample position were about 100 and $120 \mathrm{~mW}$, respectively.

The pump and Stokes beams were spatially overlapped with a dichroic mirror. These beams were introduced to an objective lens, and focused on the sample. The backward-directed SRL signal from the sample was corrected by the objective lens. The signal was separated from the pathways of the incident lights by a cubic beam splitter. After blocking the Stokes light by a shortwave pass filter, the beam was introduced to an InGaAs photodetector. The detected signal was processed by a lock-in amplifier.

The focal point of the Stokes light in the sample was arranged to the concentric circular pattern. For this arrangement, the wavefront of the Stokes light was modulated by the SLM. The image for the wavefront modulation was calculated by the iterative two-dimensional Fourier transform, as shown in Fig. 3(a). In this method, the spatial distribution of the electric field for the concentric circular focal point (A) was input. The spatial pattern of the phase (B) was random, as shown in Fig. 3(a). The set of A and B was processed by inverse Fourier transform, and the spatial distributions of the amplitude $(\mathrm{C})$ and 
(a)

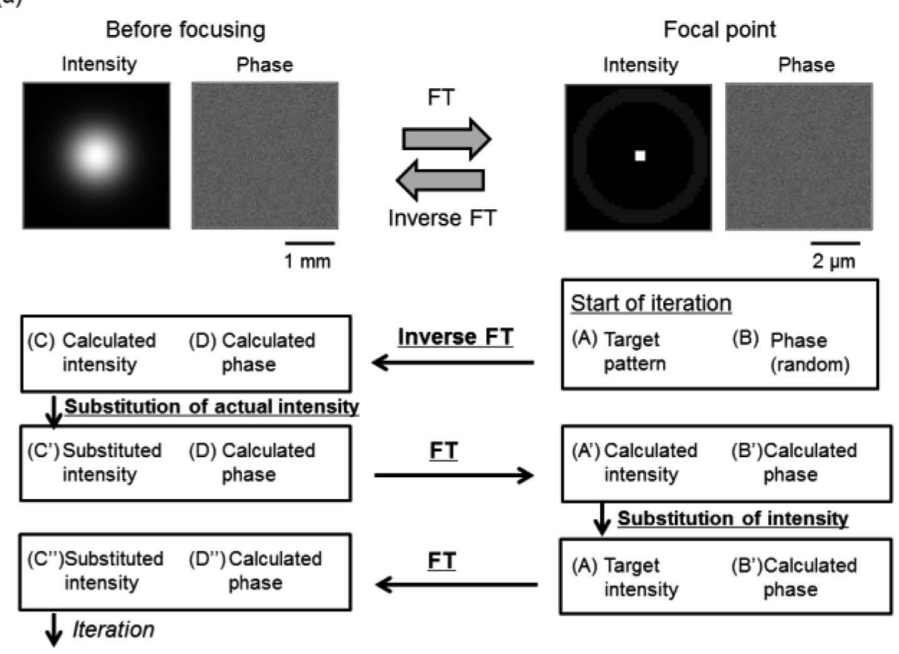

(b)
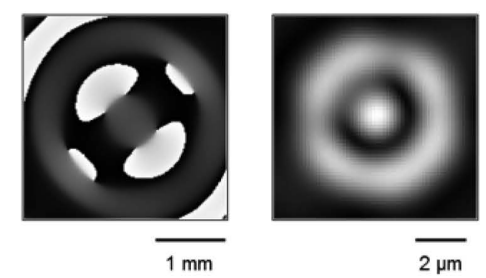

Fig. 3 (a) Scheme of the iterative Fourier transform for calculation of the wavefront pattern for the shaping of the focal point. The top four patterns represent the initial conditions of the calculation. (b) Results of the iterative Fourier transform. The wavefront of the input beam and the intensity distribution of the focal point are shown in left and right figures, respectively. For the left figure, the phase starts from black ( 0 rad.) to white $(2 \pi \mathrm{rad}$.).

phase (D) before focusing are obtained. The spatial distribution of the Stokes beam before the focusing was measured, and the actual spatial distribution was substituted to $C\left(C^{\prime}\right)$. The set of new $C^{\prime}$ and $D$ was processed by Fourier transform, and the new spatial distributions of the amplitude, $\mathrm{A}^{\prime}$, and phase, $\mathrm{B}^{\prime}$, at the focal point were obtained. The objective concentric circular distribution was again substituted to A. The new set of A and B' was again processed by inverse Fourier transform, and the spatial distributions $\mathrm{C}^{\prime \prime}$ and $\mathrm{D}^{\prime \prime}$ were renewed. The combination of the inverse Fourier and Fourier transforms was iterated 20 times. After the iteration, the obtained pattern of the wavefront was obtained as shown in Fig. 3(b). The obtained pattern shown in Fig. 3(b) was projected to the SLM, and applied for the wavefront modulation of the Stokes light.

We prepared polystyrene (PS) film with a thickness of $25 \mu \mathrm{m}$ as test samples. The incident lights were focused at the vicinity of one edge of the film. The SRS signal intensity from the PS film was measured while scanning the film along the lateral direction by a piezo stage. The spatial decay of the signal intensity by crossing the edge of the film was measured when the wavefront modulation by the SLM was on and off. The spatial decay of the signal intensity was fitted numerically under the assumption that the IRF was formulated as a Gaussian. The width of the IRF is discussed whether the SLM is on or off. For the measurements of the SRS signal from the PS film, the symmetric stretching mode of the phenyl ring with the Raman shift of $1003 \mathrm{~cm}^{-1}$ was applied. The wavelengths of the pump and Stokes lights were 1100 and $1236 \mathrm{~nm}$, respectively, where the wavenumber difference between the two lights corresponded to the Raman shift of the PS vibrational mode.

\section{Results}

Simulation of wavefront for the doughnut-shaped focal point

First, we examined the shape of the focal point by introducing the SLM. The image of the wavefront for shaping the focal point to the concentric circular shape was obtained by the iterative Fourier transform, as discussed in the Experimental
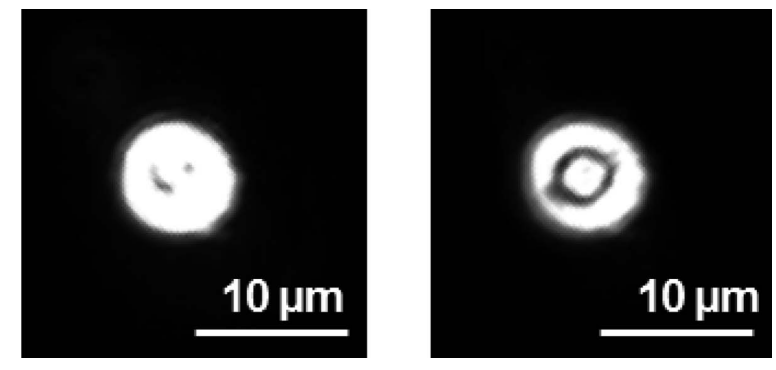

Fig. 4 Photographic images of the beam shape near the focal plane without the wavefront modulation (left) and with it (right).

section. The obtained wavefront pattern for the concentric circular shaping is shown in Fig. 3(b). The spatial distribution of the phase was projected on the SLM.

By introducing the wavefront image on the SLM, the shape of the focal point of the Stokes light was modulated. The photographic images of the Stokes light near the focal point is shown in Fig. 4. Since the diameter was too small to observe the shape in detail at the focal point, the images shown in Fig. 4 were obtained while slightly defocusing from the focal point. As shown in Fig. 4, the shape of the focal point was successfully changed from the simple round pattern to the concentric circularshaped pattern. Therefore, it is concluded that the developed algorithm for the focal point shaping works well.

\section{Improvement of IRF by focal point shaping}

We examined the IRF of the SRS microscope before and after the focal point shaping for the Stokes light. The dependence of the SRS signal from the PS film on the sample position was measured. The obtained sample position dependences of the SRS signal intensity are shown in Fig. 5. As shown in Fig. 5, the signal intensity decays at the edge of the film. To estimate the IRF from the obtained signal decay, we assume that the response function, $R(x)$, is formulated by the following Gaussian function, 
(a)

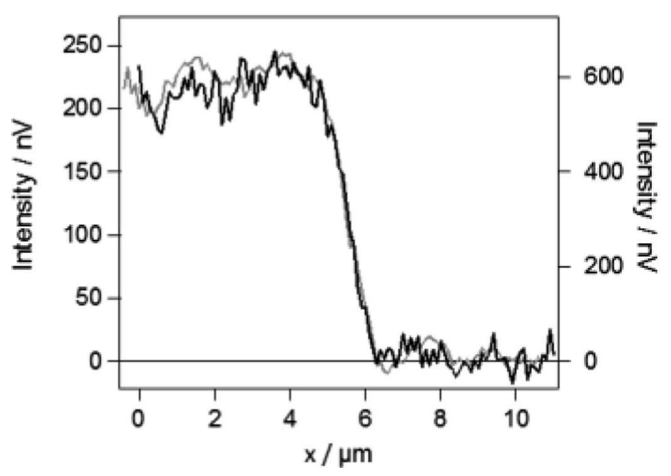

(b)

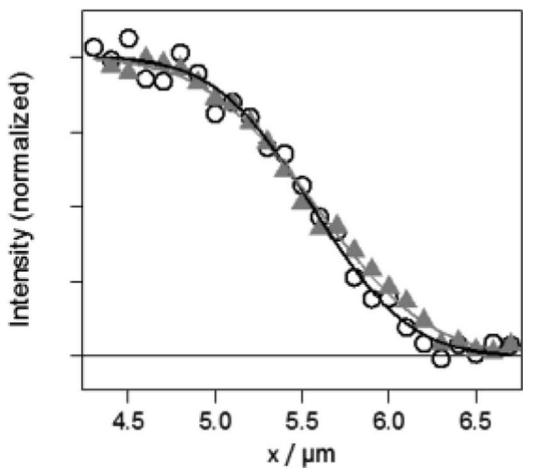

Fig. 5 Sample position dependence of the SRS signal from the PS film with the wavefront modulation (black, left axis) and without it (gray, right axis). The position around $5.5 \mu \mathrm{m}$ is enlarged in (b). In (b), the symbols represent the experimental data, and the solid lines represent the best-fitted curve with Eq. (2).

$$
R(x)=A \exp \left(-\frac{x^{2}}{\gamma^{2}}\right)
$$

where $x$ is the coordinate of the measuring point in the sample, $A$ is the amplitude of the signal, and $\gamma$ is a parameter indicating the width of the response function. The sample position dependence of the SRS signal intensity, $I(x)$, shown in Fig. 5 is fitted with the following function,

$$
I(x)=A \int \exp \left(-\frac{x^{2}}{\gamma^{2}}\right) d x,
$$

which is the integration with $x$ for Eq. (1). The results of the fitting are also shown in Fig. 5. The $\gamma$ value was estimated as $0.73 \pm 0.05$ and $0.62 \pm 0.05 \mu \mathrm{m}$, when the modulation of the wavefront by the SLM was off and on, respectively. Therefore, it is concluded that the IRF becomes narrower by about $15 \%$ due to wavefront modulation.

From the analysis, the IRF has been estimated. However, the spatial resolution is one of the most important parameters to specify a microscope. For the Gaussian function, the intensity decays to less than $2 \%$ of the peak intensity when the distance from the peak position exceeds $2 \gamma$. Therefore, when two objects are separated by a distance of more than $2 \gamma$, the two objects should be observed separately with the present microscope. We estimate the upper limit of the spatial resolution as 1.46 and $1.24 \mu \mathrm{m}$ when the SLM is off and on, respectively.

It should also be noted that the thickness of the test sample in the present study is $25 \mu \mathrm{m}$, which is longer than the coherence length for the SRS process. Therefore, the experimentally obtained IRF must be affected by the morphology of the film edge. However, the FWHM of the IRF is estimated as $1.22 \mu \mathrm{m}$ from the obtained $\gamma$ value when the SLM is off. On the other hand, from the wavelengths of the pump and Stokes beams and the NA of the objective lens, the FWHM of the signal generating area on the focal plane can be estimated at about $1.26 \mu \mathrm{m}$. This value corresponds well to the FWHM of the IRF. Therefore, the edge of the present PS film can be regarded as a step with the height of more than the spatial resolution among the axial direction, about $3 \mu \mathrm{m}$.

As mentioned above, in the present apparatus, the near-IR beams are utilized for measurements of samples buried in lightscattering media. ${ }^{18}$ However, because the near-IR beams are applied in the present apparatus, the spatial resolution is lower by a factor of about 1.5 to 3 than that of apparatuses for which visible beams are utilized. By applying the present wavefront modulation method, the reduction of the spatial resolution can be lightened without spoiling the long penetration depth in light-scattering media. The present wavefront modulation method using the SLM is an effective method to improve the IRF without changing the NA of the lens and the wavelengths of the light sources. In other words, by introducing the wavefront modulation system, the IRF of a nonlinear spectroscopic microscope can be further narrowed.

\section{Discussion}

As shown above, the width of the IRF became narrower by the introduction of the SLM to the SRS microscope. It is important to estimate how the IRF can become narrow by the SLM. In this section, we estimate the IRF by numerical calculations.

First, we estimate the IRF without the SLM. The shape of the focal spot is estimated by the Fourier transform of the spot shape of the incident beam with the diameter of the incident beam, the NA of the objective lens, and the wavelength. Here, the diameter of the incident beam exhibited the Gaussian-like shape with the full width at half maximum (FWHM) of $1.2 \mathrm{~mm}$. The NA of the objective lens was 0.65 , and the wavelength was $1236 \mathrm{~nm}$. Under the condition, the shape of the focal spot was estimated as shown in Fig. 6(a). The spot exhibits a twodimensional Gaussian shape with the FWHM of $2.18 \mu \mathrm{m}$. When the pump beam is also focused with the same objective lens, the spot shape of the focal point is also a Gaussian function. The SRS signal generates from the area estimated by multiplying the spot shapes of the pump and Stokes beams, as shown in Fig. 6(b). From this result, the FWHM of the area generating the SRS signal is $1.54 \mu \mathrm{m}$, without the SLM.

When the SLM is installed, and the pattern of the wavefront is modulated as shown in Fig. 3(a), the spot shape of the Stokes light at the focal point is changed to the concentric circles, as shown in Fig. 3(b). In this situation, the FWHM of the center circle is reduced to $1.83 \mu \mathrm{m}$ from the original Gaussian shape, $2.18 \mu \mathrm{m}$, as shown in Fig. 6(c). The area generating the SRS signal is calculated by multiplying the modulated pattern of the Stokes light and the original pump light as shown in Fig. 6(d). As shown in Fig. 6(d), the FWHM of the center circle generating the strongest SRS signal is $1.41 \mu \mathrm{m}$. Note that the intensities of the side bands appearing in Fig. 6(d) are less by a factor of $10 \%$ than the main band. Therefore, the contributions from the side 
(a)

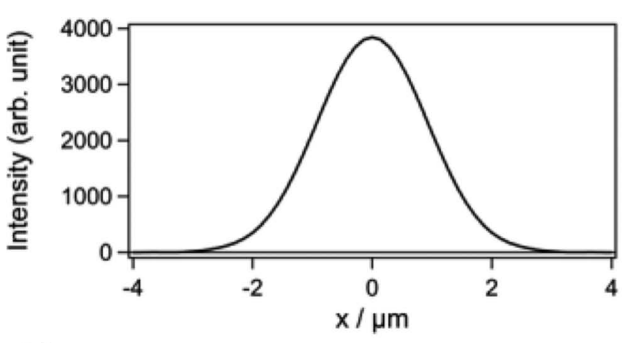

(c)

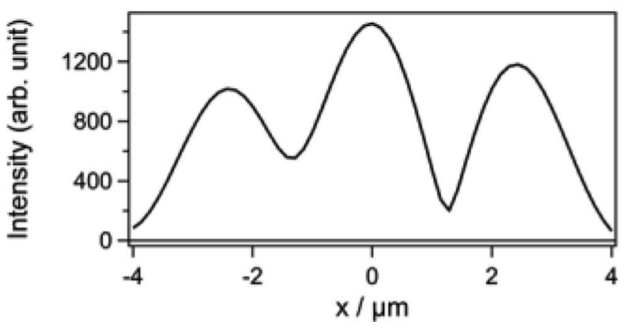

(b)

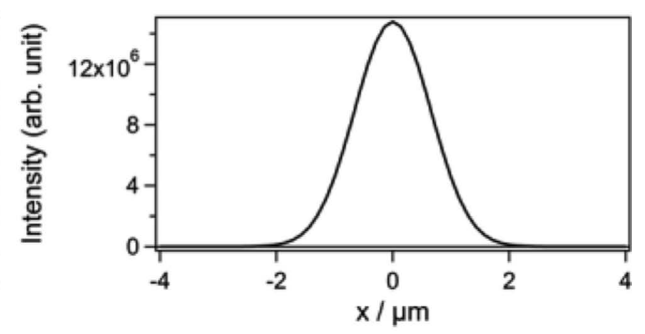

(d)

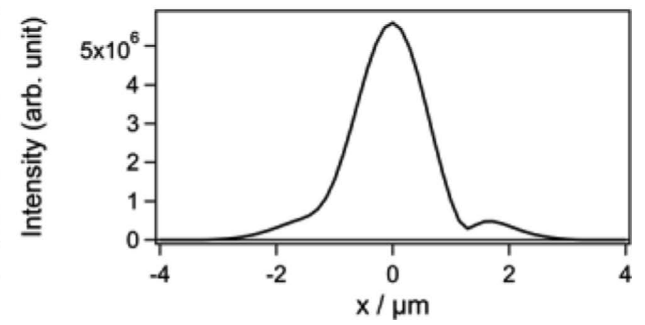

Fig. 6 Simulated spatial distribution of the electric field intensity and the SRS signal intensity along the $X$ (longitudinal) axis. The position along the $Y$ axis is fixed to the center of the pattern $(Y=0)$. (a) Electric field intensity for the Stokes beam without the wavefront modulation. (b) SRS signal intensity without the wavefront modulation. (c) Electric field intensity for the Stokes beam with the wavefront modulation. (d) SRS signal intensity with the wavefront modulation.

bands can be considered as smaller than the experimental uncertainties. Therefore, the width of the area generating the SRS signal is estimated to be narrowed by $9 \%$ by introducing the SLM. As discussed above, the experimentally-estimated IRF is narrowed by $15 \%$ by introducing the SLM. Therefore, the IRF narrowing by introducing the SLM can be well estimated by the numerical simulation. The width of the IRF has become a controllable parameter by the introduction of the SLM.

It is expected that the width of the IRF should be narrowed more by changing the focal point to another pattern. We simulated a triple concentric circular pattern as shown in Fig. 7. As shown in Fig. 7, the width of the center circle for the triple concentric circular pattern is less by a factor of $9 \%$ than that for the double concentric pattern. Therefore, it is probable that the IRF should be narrowed more by changing the focal spot to a more complicated pattern. However, it should also be noted that the signal intensity becomes smaller as the pattern of the focal spot becomes more complicated. As shown in Fig. 7, the amplitude of the center circle for the triple concentric circular pattern decreases by $26 \%$ from that for the double concentric circular pattern. To apply the wavefront modulation method more effectively, it is important to select the focal spot pattern to optimize the balance between the width of the IRF and the signal intensity, namely, the signal-to-noise ratio for the measurements.

It should be noted that the simulated patterns shown in Figs. 6 and 7 are asymmetric, although the input patterns are all symmetric. At the present condition, the FWHM of the incident beam diameter was $1.2 \mathrm{~mm}$, and only $60 \times 60$ pixels in the SLM were used for the wavefront modulation. Due to the low number of the pixels, the simulated beam pattern may be distorted in the numerical simulation procedure, resulting in the asymmetric properties. When beam expanders are introduced to the apparatus and more SLM pixels are utilized, the asymmetric properties should be reduced.

At the present stage, the improvement of the spatial resolution along the axial direction is not examined in detail, although it is

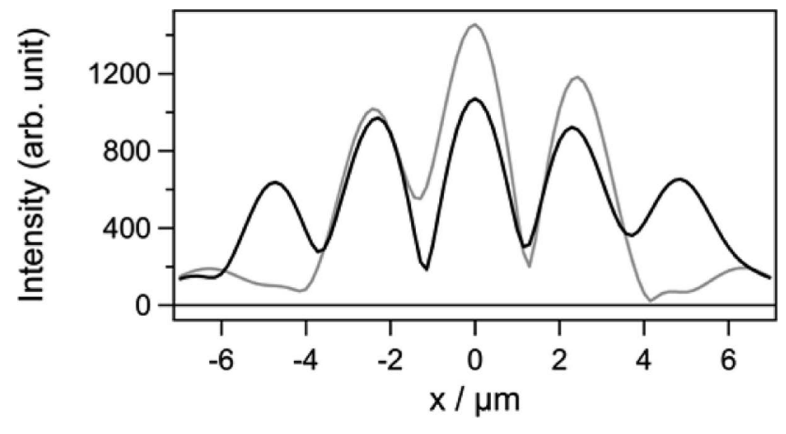

Fig. 7 Simulated spatial distribution of the electric field intensity along the $X$ axis for the double (gray) and triple (black) concentric circular patterns.

another important property to specify the microscope. At the present stage, we consider that the resolution should not be largely changed by the introduction of the SLM because the wavelengths of the beams and the NA of the objective lens are not changed. However, we will estimate quantitatively the signal intensity profile along the axial direction, and will try to improve the resolution in the near future.

\section{Conclusion}

To develop a vibrational spectroscopic microscopy, we have introduced an SLM to an SRS microscope. In the developed apparatus, the wavefront of the Stokes beam can be modulated to any shape. We modulated the wavefront to make the shape at the focal point of an objective lens concentric circles. By combining the wavefront modulation and SRS spectroscopy, we attempt to improve the IRF of the microscope. The wavefront pattern was calculated by utilizing the iterative Fourier transform 
method. As a result, the spot shape at the focal point for the Stokes light was successfully changed to the concentric circles. When the IRF of the SRS microscope was estimated by applying to the edge of a PS film, the IRF was narrowed by about $15 \%$, as estimated from the numerical simulation. From this result, it is suggested that combining the wavefront modulation and nonlinear vibrational spectroscopy is a way to improve the IRF of fully optical vibrational spectroscopic microscopes.

\section{References}

1. K. Konig, J. Microsc., 2000, 200, 83.

2. I. W. Levin and R. Bhargava, Annu. Rev. Phys. Chem., 2005, 56, 429.

3. B. N. G. Giepmans, S. R. Adams, M. H. Ellisman, and R. Y. Tsien, Science, 2006, 312, 217.

4. W. L. Chan, J. Deibel, and D. M. Mittleman, Rep. Prog. Phys., 2007, 70, 1325.

5. J. X. Cheng and X. S. Xie, "Coherent Raman Scattering Microscopy", 2013, CRC Press, Boca Raton.

6. P. Lasch and D. Naumann, Biochim. Biophys. Acta, 2006, $1758,814$.

7. E. Hecht, "Optics", 4th ed., 2002, Pearson Education Inc.,
New York.

8. A. Zumbusch, G. R. Holtom, and X. S. Xie, Phys. Rev. Lett., 1999, 82, 4142.

9. C. J. Frank, D. C. B. Redd, T. S. Gansler, and R. L. McCreery, Anal. Chem., 1994, 66, 319.

10. J. W. Goodman, "Introduction to Fourier Optics", 3rd ed., 2005, Roberts \& Company Publishers, Greenwood Village.

11. S. W. Hell and J. Wichmann, Opt. Lett., 1994, 19, 780.

12. A. M. H. Wong and G. V. Eleftheriades, Sci. Rep., 2013, 3, 1.

13. T. Watanabe, Y. Iketaki, T. Omatsu, K. Yamamoto, S. Ishiuchi, M. Sakai, and M. Fujii, Chem. Phys. Lett., 2003, 371, 634 .

14. J.-Y. Lin, R.-P. Huang, P.-S. Tsai, and C.-H. Lee, J. Opt. A: Pure Appl. Opt., 2009, 11, 015301.

15. J. Tang, R. N. Germain, and M. Cui, Proc. Natl. Acad. Sci. U. S. A., 2012, 109, 8434.

16. J. R. Allen, S. T. Ross, and M. W. Davidson, ChemPhysChem, 2014, 15, 566.

17. P. Wang, M. N. Slipchenko, J. Mitchell, C. Yang, E. O. Potma, X. Xu, and J.-X. Cheng, Nat. Photon., 2013, 7, 449.

18. M. Banno and H. Yui, Appl. Spectrosc., in press.

19. C. L. Evans and X. S. Xie, Annu. Rev. Anal. Chem., 2008, 1, 883 . 